PROCEEDINGS OF THE

AMERICAN MATHEMATICAL SOCIETY

Volume 125, Number 1, January 1997, Pages 67-74

S 0002-9939(97)03663-0

\title{
PRIME IDEALS IN POLYNOMIAL RINGS IN SEVERAL INDETERMINATES
}

\author{
MIGUEL FERRERO
}

(Communicated by Ken Goodearl)

\begin{abstract}
If $P$ is a prime ideal of a polynomial ring $K[x]$, where $K$ is a field, then $P$ is determined by an irreducible polynomial in $K[x]$. The purpose of this paper is to show that any prime ideal of a polynomial ring in $n$-indeterminates over a not necessarily commutative ring $R$ is determined by its intersection with $R$ plus $n$ polynomials.
\end{abstract}

\section{INTRODUCTION}

Let $K$ be a field and $K[x]$ the polynomial ring over $K$ in an indeterminate $x$. If $P$ is a prime ideal of $K[x]$, then there exists an irreducible polynomial $f$ in $K[x]$ such that $P=K[x] f$. This result is quite old and basic; however no corresponding result seems to be known for a polynomial ring in $n$ indeterminates $x_{1}, \ldots, x_{n}$ over $K$. Actually, it seems to be very difficult to find some system of generators for a prime ideal of $K\left[x_{1}, \ldots, x_{n}\right]$.

Now, $K\left[x_{1}, \ldots, x_{n}\right]$ is a Noetherian ring and by a converse of the principal ideal theorem for every prime ideal $P$ of $K\left[x_{1}, \ldots, x_{n}\right]$ there exist $n$ polynomials $f_{1}, \ldots, f_{n}$ such that $P$ is minimal over $\left(f_{1}, \ldots, f_{n}\right)$, the ideal generated by $\left\{f_{1}, \ldots, f_{n}\right\}$ ([4], Theorem 153). Also, as a consequence of ([1], Theorem 1) it follows that any prime ideal of $K\left[x_{1}, \ldots, x_{n}\right]$ is determined by $n$ polynomials. However it is not clear in [1] how to find these polynomials and no converse result is proved. The purpose of this paper is to show a result which in particular implies that every prime ideal of $K\left[x_{1}, \ldots, x_{n}\right]$ is determined by a sequence of $n$ polynomials which is in some sense irreducible, and such that the converse is also true.

In general, let $R$ be any prime ring. If $P$ is a nonzero prime ideal of the polynomial ring $R[x]$ with $P \cap R=0$ (an $R$-disjoint prime ideal), then $P=Q[x] f_{0} \cap R[x]$ where $Q$ is a ring of right quotients of $R$ and $f_{0} \in C[x]$ is an irreducible polynomial, $C$ being the extended centroid of $R$ ([2], Corollary 2.7). This characterization has the problem that we have to compute $Q$ to have a prime ideal determined. A better way (an intrinsic one) to determine an $R$-disjoint prime ideal has been given in [3]. We proved that every $R$-disjoint prime ideal $P$ of $R[x]$ is determined by just one polynomial in $P$ which is in some sense irreducible ([3], Theorem 1.4).

Received by the editors March 15, 1995 and, in revised form, July 28, 1995.

1991 Mathematics Subject Classification. Primary 16D30, 16S36; Secondary 12E05.

This research was supported by a grant given by Conselho Nacional de Desenvolvimento Científico e Tecnológico (CNPq), Brazil.

(C) 1997 American Mathematical Society 
The purpose of this paper is to extend the above result. We show that any prime ideal $P$ in a polynomial ring in $n$ indeterminates is determined by its intersection with $R$ plus $n$ polynomials in $P$. Moreover, these polynomials define a sequence which has some irreducibility property and will be called completely irreducible $(\bmod P \cap R)$. The converse is also true, i.e., for any prime ideal $Q$ of $R$ and any completely irreducible sequence $(\bmod Q)$, there exists a unique prime ideal determined by them. It turns out that there exists a one-to-one correspondence between prime ideals of $R\left[x_{1}, \ldots, x_{n}\right]$ and equivalence classes of tuples $\left(Q, f_{1}, \ldots, f_{n}\right)$, where $Q$ is a prime ideal of $R$ and $\left(f_{1}, \ldots, f_{n}\right)$ is a completely irreducible sequence $(\bmod Q)$.

Throughout this paper $R$ is any ring with an identity element. If $f \in R[x]$, then $\delta f$ (resp. $l c(f))$ denotes the degree (resp. leading coefficient) of $f$. The leading coefficient of the zero polynomial will be defined as the identity of $R$. Finally, the notation $\supset$ means strict inclusion.

\section{Prime ideals in $R[x]$}

Throughout this section $Q$ denotes a prime ideal of $R$. We will describe all the prime ideals $P$ of $R[x]$ such that $P \cap R=Q$. The results can be obtained by factoring out from $R$ and $R[x]$ the ideals $Q$ and $Q[x]$, respectively, and then applying the results of [2] and [3]. However we will give here direct arguments for the sake of completeness.

We define

$$
\Gamma_{Q}=\{f \in R[x]: \operatorname{arf}-f r a \in Q[x] \text {, for all } r \in R, \delta f \neq 0 \text {, and } a=l c(f) \notin Q\} .
$$

The definition of $\Gamma_{Q}$ intends to give an extension of the definition of $\Gamma_{R}$ in [2], Section 1. Thus they should coincide when $Q=0$. However they are slightly different. In fact, we exclude here nonzero constant polynomials. Also, the convention $l c(0)=1$ allows us to assume always that $l c(f) \notin Q$. Note that the zero polynomial belongs to $\Gamma_{Q}$.

For $f \in \Gamma_{Q}$ with $a=l c(f)$, define

$$
[Q, f]=\left\{g \in R[x]: \text { there exists } e \geq 0 \text { with } g(R a)^{e} \subseteq R[x] f+Q[x]\right\} .
$$

We can easily see that $[Q, f]$ is an ideal of $R[x]$ containing $f$ and $Q[x]$.

In the rest of the section we denote by $a$ and $b$ the leading coefficients of $f$ and $g$, respectively. We begin with the following.

Lemma 1.1 (cf. [2, Lemma 1.1]). Assume that $f \in \Gamma_{Q}$. If $g \in[Q, f]$ and $\delta g<\delta f$, then $g \in Q[x]$. In particular, $[Q, f] \cap R=Q$.

Proof. Note that $a \notin Q$. So by assumption, there exists an ideal $H \nsubseteq Q$ of $R$ such that for every $r \in H$ there is $k=x^{m} b_{m}+\ldots+b_{0} \in R[x]$ with $g r a=k f+h$, where $h \in Q[x]$. We have $b_{m} a \in Q$ and for $s \in R$, grasa $=k f s a+h s a=k a s f+h s a+l$, for some $l \in Q[x]$.

Assume, by induction, that $b_{i} a \in Q$ for $i=m, \ldots, m-t+1$. Then from the above relation we get $b_{m-t} a s a \in Q$, for every $s \in R$. It follows that $b_{m-t} a \in Q$ because $a \notin Q$. Hence $b_{i} a \in Q$ for $i=1, \ldots, m$, and so $g H a R a \subseteq Q[x]$. Thus $g \in Q[x]$ since $Q[x]$ is a prime ideal of $R[x]$. The proof is complete. 
Note that the definition $[f]_{R}$ in [2] also looks different than our definition here of $[0, f]$, for $f \in \Gamma_{0}$. However we see they coincide. In fact, put

$$
I=\{g \in R[x] \text { : there exists } H \triangleleft R, H \nsubseteq \mathbb{Q} \text {, such that } g H a \subseteq R[x] f+Q[x]\} .
$$

Lemma 1.2. $[Q, f]=I$.

Proof. If $f=0$, then $[Q, f]=Q[x]$ as well as $I=Q[x]$, since $Q[x]$ is a prime ideal. In general, it is clear that $[Q, f] \subseteq I$. Assume that $g \in I, \delta g=m$. If $m<\delta f=n$ we prove as in Lemma 1.1 that $g \in Q[x] \subseteq[Q, f]$. Suppose $\delta g=n$. We have $g r a-b r f \in I$, for any $r \in R$, and $\delta(g r a-b r f)<n$. Hence gra $-b r f \in Q[x]$ and it follows that $g R a \subseteq R[x] f+Q[x]$. Finally, assume by induction that $m>n$ and for any $h \in I$ with $\delta h<m$ we have $h(R a)^{m-n} \subseteq R[x] f+Q[x]$. For $r \in R$ put $l=g r a-x^{m-n} r b f \in I$. Applying the induction assumption to $l$ we easily get $g(R a)^{m-n+1} \subseteq R[x] f+Q[x]$. The result follows.

Now we prove the following key result.

Proposition 1.3. Assume that $f, g \in \Gamma_{Q}$ and $g \in[Q, f]$. Then $[Q, g] \subseteq[Q, f]$. Also, $[Q, g]=[Q, f]$ if and only if $\delta g=\delta f$.

Proof. Take $h \in[Q, g]$. By Lemma 1.2 there exists an ideal $H^{\prime}$ of $R$ with $H^{\prime} \nsubseteq Q$ such that $h H^{\prime} b \subseteq R[x] g+Q[x]$. Also, by assumption there exists $H \triangleleft R$ with $H \nsubseteq Q$ and $g H a \subseteq R[x] f+Q[x]$. Thus $h H^{\prime} b H a \subseteq R[x] f+Q[x]$ and it follows that $h \in[Q, f]$ since $H^{\prime} b H \nsubseteq Q$. The first part follows.

Assume $\delta g=\delta f$. For every $r \in R, f r b-\arg \in[Q, f]$ and $\delta(f r b-a r g)<n$. Then $f r b-\arg \in Q[x]$ by Lemma 1.1. Therefore $f R b \subseteq R[x] g+Q[x]$ and thus $f \in[Q, g]$. Using the first part we get $[Q, g]=[Q, f]$.

The converse follows easily from Lemma 1.1 , since $f, g \notin Q[x]$.

Remark 1.4. We can define $[Q, f]$ in a dual way. In fact, denote by $[Q, f]^{\prime}$ the ideal of $R[x]$ defined as $[Q, f]$, but with the condition $(a R)^{e} g \subseteq f R[x]+Q[x]$ instead of the condition $g(R a)^{e} \subseteq R[x] f+Q[x]$. As in Lemma 1.1 we can prove that if $g \in[Q, f]^{\prime}$ and $\delta g<\delta f$, then $g \in Q[x]$. Actually, it is not hard to show that $[Q, f]=[Q, f]^{\prime}$.

Corollary 1.5 (cf. [2, Corollary 1.3]). Assume that $f, g \in \Gamma_{Q}$. Then $[Q, f]=$ $[Q, g]$ if and only if gra - br $f \in Q[x]$ for every $r \in R$.

Proof. If $[Q, f]=[Q, g]$ we have $\delta f=\delta g$ by Proposition 1.3 . Hence we easily obtain gra $-b r f \in Q[x]$ for all $r \in R$. Conversely, if $\operatorname{gra}-\operatorname{brf} \in Q[x]$, for every $r \in R$, we have $g R a \subseteq R[x] f+Q[x]$ and so $g \in[Q, f]$. In particular, $\delta g \geq \delta f$ since $g \notin Q[x]$. Also, $b R f \subseteq g R[x]+Q[x]$ and it follows that $f \in[Q, g]^{\prime}$. Hence $\delta f \geq \delta g$ by Remark 1.4. It follows that $\delta f=\delta g$ and Proposition 1.3 gives $[Q, f]=[Q, g]$.

The following is well-known (or can easily be obtained by factoring out $Q$ and $Q[x]$ from $R$ and $R[x]$, respectively).

Lemma 1.6. Let $P$ be an ideal of $R[x]$. Then $P$ is prime if and only if $P \cap R$ is a prime ideal of $R$ and either $P=(P \cap R)[x]$ or $P$ is maximal amongst ideals $I$ of $R[x]$ such that $I \cap R=P \cap R$.

Now we extend the definition of $\Gamma$-completely irreducibility (see [3], Definition $1.2)$. 
Definition 1.7. We say that a polynomial $f \in \Gamma_{Q}$ is $\Gamma_{Q}$-completely irreducible if the following condition is satisfied:

If there exist $b \in R, g \in \Gamma_{Q}$, and $h \in R[x]$ such that $f b \notin Q[x]$ and $f b-h g \in Q[x]$, then $\delta g=\delta f$.

Note that the above definition is vacuously true for $f=0$. So we may consider the zero polynomial as a $\Gamma_{Q}$-completely irreducible polynomial.

Remark 1.8. Assume that $f \in \Gamma_{Q}$. Then we can easily see that if $b \notin Q$ we have $f b \in \Gamma_{Q}$ and $[Q, f]=[Q, f b]$ (use Proposition 1.3).

Now we can prove the main result of this section.

Theorem 1.9 (cf. [3, Theorem 1.4]). Let $R$ be any ring. Then an ideal $P$ of $R[x]$ is prime if and only if $Q=P \cap R$ is prime and there exists a $\Gamma_{Q}$-completely irreducible polynomial $f \in R[x]$ such that $P=[Q, f]$. Moreover, the polynomial $f$ can be chosen to be any polynomial in $P$ which is of minimal degree with respect to the condition $l c(f) \notin Q$.

Proof. If $P$ is a prime ideal, then $Q=P \cap R$ is prime. In case $P=Q[x]$ we take $f=0$ and so $P=[Q, f]$ follows. Assume $P \supset Q[x]$ and take any polynomial $f \in P$ of minimal degree $n$ with respect to the condition $a=l c(f) \notin Q$. For any $r \in R$, fra $-\operatorname{arf} \in P$ and $\delta(f r a-a r f)<n$. It follows that $f \in \Gamma_{Q}$. We show that $P=[Q, f]$.

Suppose $g \in P, \delta g=m$. If $m<n$ we have $g \in Q[x]$ by minimality of $n$. Repeating the arguments of the proof of Lemma 1.2 we show, by induction, that $g \in[Q, f]$. Consequently $P \subseteq[Q, f]$ and Lemma 1.6 gives $P=[Q, f]$.

Assume that for some $b \in R, g \in \Gamma_{Q}$, and $h \in R[x]$ we have $f b \notin Q[x]$ and $f b-h g \in Q[x]$. By Remark 1.8 and Proposition 1.3 we have $P=[Q, f]=[Q, f b] \subseteq$ $[Q, g]$. It follows that $[Q, f]=[Q, g]$ by maximality of $P$ and hence $\delta f=\delta g$. Consequently $f$ if $\Gamma_{Q}$-completely irreducible.

Conversely, assume that $Q$ is prime and $P=[Q, f]$ for some $f \in \Gamma_{Q}$ which is $\Gamma_{Q}$-completely irreducible. If $f=0$ we clearly have $P=Q[x]$ and so $P$ is prime. Suppose $f \neq 0$ and take an ideal $M$ of $R[x]$ which is maximal with respect to the conditions $M \supseteq P$ and $M \cap R=Q$. Then $M$ is prime and it follows from the first part that $M=[Q, g]$ for some $g \in \Gamma_{Q}$. Since $f \in P$ there exists $e \geq 0$ with $f(R b)^{e} \subseteq R[x] g+Q[x]$. Using now the irreducibility property of $f$ we easily obtain that $\delta f=\delta g$ and consequently $P=M$, by Proposition 1.3. Hence $P$ is a prime ideal and the proof is complete.

Let $\mathcal{P}$ be the set of all the pairs $(Q, f)$ where $Q$ is a prime ideal of $R$ and $f \in \Gamma_{Q}$ is a $\Gamma_{Q}$-completely irreducible polynomial. We define an equivalence relation by saying that $(Q, f) \sim\left(Q^{\prime}, g\right)$ if and only if $Q=Q^{\prime}$ and $f r b-\arg \in Q[x]$, for every $r \in R$, where $a=l c(f)$ and $b=l c(g)$. By Corollary 1.5, $(Q, f) \sim\left(Q^{\prime}, g\right)$ if and only if $[Q, f]=\left[Q^{\prime}, g\right]$. We clearly have

Theorem 1.10. Let $R$ be any ring and let $\mathcal{P}$ be the set of all the pairs as above. Then the mapping sending $(Q, f)$ to $[Q, f]$ defines a one-to-one correspondence between the set of all the equivalence classes in $\mathcal{P}$ and the set of all the prime ideals of $R[x]$. 


\section{Prime ideals in $R\left[x_{1}, \ldots, x_{n}\right]$}

The purpose of this section is to extend the results of the former one. We prove similar results for a polynomial ring in $n$-indeterminates. We begin with a technical lemma.

Let $R$ be a ring, $T=R\left[x_{1}, \ldots, x_{n}\right]$ the polynomial ring over $R$ in $n$-indeterminates $x_{1}, \ldots, x_{n}$, and $S=T[t]$ the polynomial ring over $T$ in an indeterminate $t$.

If $f \in S$ we consider $f$ as a polynomial in $t$ with coefficients in $T$ and we denote by $\delta f$ (resp. a) the degree (resp. leading coefficient) of $f$ with respect to $t$.

For a polynomial $h \in T, \Delta h$ denotes the total degree of $h$ as a polynomial in the indeterminates $x_{1}, \ldots, x_{n}$. Also, for $g=a_{m} t^{m}+\ldots+a_{0} \in S, a_{j} \in T$ for $0 \leq j \leq m$, $\Delta g$ is defined as $\max \left\{\Delta a_{0}, \ldots, \Delta a_{m}\right\}$.

Let $P$ be a prime ideal of $S$ and $Q=P \cap T$. Assume that $P \supset Q[t]$ and $f \in P$ is a polynomial of minimal degree $u$ with respect to the condition $a=l c(f) \notin Q$. Put $\Delta f=v$.

In the next lemma $b$ denotes the leading coefficient of $g \in S$ and $(f)$ the ideal of $S$ generated by $f$. We have

Lemma 2.1. Under the same notation as above, given an integer $m \geq u$, for every $g \in P$ with $\delta g \leq m$, and for every $r_{0}, r_{1}, \ldots, r_{m-n}$ in $R$, there exists $h \in(f)$ such that $l=g r_{0} a r_{1} a \ldots r_{m-n} a-h \in Q[t]$ and $\Delta l \leq w+(m-u+1) v$, where $w=\Delta g$.

Proof. If $m=u$ and $\delta g<u$ we have $g \in Q[t]$. So it is enough to take $h=0$ in this case. If $\delta g=u$, then gra - brf $f \in[t]$, for every $r \in R$, since $g r a-b r f \in P$ and $\delta(g r a-b r f)<u$. Also $\Delta(g r a-b r f) \leq \max \{\Delta(g r a), \Delta(b r f)\} \leq w+v$.

By induction, assume that $m>u$ and the result is true for any polynomial $p \in P$ with $\delta p \leq m-1$. Let $g$ be a polynomial of degree $m$ in $P$ with $\Delta g=$ $w$. For $r_{0} \in R$ put $g_{r_{0}}=g r_{0} a-t^{m-u} b r_{0} f \in P$. Then $\delta g_{r_{0}}<m$ and $\Delta g_{r_{0}} \leq$ $\max \left\{\Delta\left(g r_{0} a\right), \Delta\left(t^{m-u} b r_{0} f\right)\right\} \leq w+v$.

Therefore, for any $r_{1}, \ldots, r_{m-n} \in R$ there exists $h \in(f)$ such that

$$
l=g_{r_{0}} r_{1} a \ldots r_{m-n} a-h \in Q[t]
$$

and

$$
\Delta l \leq w+v+(m-u) v=w+(m-u+1) v
$$

Hence $g r_{0} a r_{1} a \ldots r_{m-n} a-h^{\prime} \in Q[t]$, where $h^{\prime}=h+t^{m-u} b r_{0} f r_{1} a \ldots r_{m-n} a \in(f)$, and the proof is complete.

For a subset $B \subset S, \delta B($ resp. $\Delta B)$ denotes $\sup \{\delta h: h \in B\}($ resp. $\sup \{\Delta h$ : $h \in B\})$. The following is clear.

Corollary 2.2. Under the same notation as above, if $B \subseteq P, \delta B \leq m$, and $\Delta B \leq$ $w$, for every $h \in B(R a)^{m-u+1}$ there exists $h^{\prime} \in(f)$ such that $h-h^{\prime} \in Q[t]$ and $\Delta\left(h-h^{\prime}\right) \leq w+(m-u+1) v$.

Now we change notations. Let $R$ be any ring and $S=R\left[x_{1}, \ldots, x_{n}\right]$ a polynomial ring over $R$ in $n$ indeterminates $x_{1}, \ldots, x_{n}$.

We fix the natural order in the variables. Denote by $R_{i}$ the polynomial ring $R\left[x_{1}, \ldots, x_{i}\right]$. If $f_{i} \in R_{i}$ we consider $f_{i}$ as a polynomial in $x_{i}$ with coefficients in $R_{i-1}$ and denote by $\delta f_{i}$ (resp. $a_{i}$ ) its degree (resp. leading coefficient) with respect to $x_{i}$. Also, $\Delta_{i} f_{i}$ denotes the total degree of $f_{i}$ as a polynomial in $x_{1}, \ldots, x_{i}$.

Let $Q$ be a prime ideal of $R$ and put $Q[x]=Q\left[x_{1}, \ldots, x_{n}\right]$. We denote by $\mathcal{F}$ (resp. $\left.\mathcal{F}_{i}\right)$ the set of all the sequences $\left(f_{1}, \ldots, f_{n}\right)\left(\operatorname{resp} .\left(f_{1}, \ldots, f_{i}\right)\right)$, where $f_{j} \in R_{j}$, 
$1 \leq j \leq n$. If $f=\left(f_{1}, \ldots\right)$ is a sequence in $\mathcal{F}\left(\right.$ resp. $\left.\mathcal{F}_{i}\right)$, then $(f)\left(\right.$ resp. $\left.(f)_{i}\right)$ denotes the ideal of $S$ (resp. $R_{i}$ ) generated by $\left\{f_{1}, \ldots, f_{n}\right\}$ (resp. $\left\{f_{1}, \ldots, f_{i}\right\}$ ). For $f \in \mathcal{F}$ we put

$$
\begin{aligned}
{\left[Q, f_{1}, \ldots, f_{n}\right]=} & \left\{g \in S: \text { there exist } e_{i} \geq 0,1 \leq i \leq n,\right. \text { such that } \\
& \left.g\left(R a_{n}\right)^{e_{n}} \ldots\left(R a_{1}\right)^{e_{1}} \subseteq Q[x]+(f)\right\} .
\end{aligned}
$$

Note that if $g\left(R a_{n}\right)^{e_{n}} \ldots\left(R a_{1}\right)^{e_{1}} \subseteq Q[x]+(f)$, then $g\left(R a_{n}\right)^{d_{n}} \ldots\left(R a_{1}\right)^{d_{1}} \subseteq Q[x]+$ $(f)$, for every sequence of integers $\left(d_{j}\right)_{1 \leq j \leq n}$ with $d_{i} \geq e_{i}$ for $1 \leq i \leq n$. Then it follows easily that $\left[Q, f_{1}, \ldots, f_{n}\right]$ is an ideal of $S$.

Now we can prove the main result of this paper. It shows that every prime ideal of $S$ is completely determined by its intersection with $R$ plus $n$ polynomials in $S$.

Theorem 2.3. Let $P$ be a prime ideal of $S$ with $P \cap R=Q$. Then there exist $n$ polynomials $f_{1}, \ldots, f_{n}$ in $S$ such that $\left(f_{1}, \ldots, f_{n}\right) \in \mathcal{F}$ and $P=\left[Q, f_{1}, \ldots, f_{n}\right]$.

Proof. Put $P_{i}=P \cap R_{i}, 1 \leq i \leq n$. If $P_{1}=Q\left[x_{1}\right]$ we take $f_{1}=0\left(\right.$ so $\left.a_{1}=1\right)$. If $P_{1} \supset Q\left[x_{1}\right]$, take a polynomial $f_{1}$ of minimal degree in $P_{1}$ with respect to the condition $a_{1} \notin Q$. It follows that $P_{1}=\left[Q, f_{1}\right]$, by Theorem 1.9. Moreover, if $B \subseteq P_{1}$ and $\delta B \leq m$ for some integer $m$, then there exists $e_{1} \geq 0$ such that $B\left(R a_{1}\right)^{e_{1}} \subseteq Q\left[x_{1}\right]+(f)_{1}$, by Corollary 2.2 (with $n=0$ and $t=x_{1}$ ).

We continue the construction by induction. Assume there exists a sequence $\left(f_{1}, \ldots, f_{i-1}\right) \in \mathcal{F}_{i-1}$ such that $P_{i-1}=\left[Q, f_{1}, \ldots, f_{i-1}\right]$. Moreover, if $B \subseteq P_{i-1}$ and $\Delta_{i-1} B \leq m$ for some integer $m$, there exist $e_{1}, \ldots, e_{i-1} \geq 0$ such that

$$
B\left(R a_{i-1}\right)^{e_{i-1}} \ldots\left(R a_{1}\right)^{e_{1}} \subseteq Q\left[x_{1}, \ldots, x_{i-1}\right]+(f)_{i-1} .
$$

If $P_{i}=P_{i-1}\left[x_{i}\right]$ we take $f_{i}=0$. If $P_{i} \supset P_{i-1}\left[x_{i}\right]$ we take a polynomial $f_{i} \in P_{i}$ of minimal degree with respect to the condition $a_{i} \notin P_{i-1}$. Applying again Theorem 1.9 we have $P_{i}=\left[P_{i-1}, f_{i}\right]$. It remains to show that $\left[Q, f_{1}, \ldots, f_{i}\right]=P_{i}$ and complete the proof of the induction argument.

If $g \in\left[Q, f_{1}, \ldots, f_{i}\right]$, then $g\left(R a_{i}\right)^{e_{i}} \ldots\left(R a_{1}\right)^{e_{1}} \subseteq Q\left[x_{1}, \ldots, x_{i}\right]+(f)_{i} \subseteq P_{i}$, for some integers $e_{1}, \ldots, e_{i} \geq 0$. Since $P_{i}$ is prime and $a_{j} \notin P_{i}$, for $1 \leq j \leq i$, it follows that $g \in P_{i}$.

Conversely, assume that $C \subseteq P_{i}$ and $\Delta_{i} C \leq m$. Then we have $\delta C \leq m$ and $\Delta_{i-1} C \leq m$. By Corollary 2.2 there is an integer $l \geq 0$ such that for every $h \in C\left(R a_{i}\right)^{m-u_{i}+1}$ there exists $h^{\prime} \in\left(f_{i}\right)$ with $h-h^{\prime} \in P_{i-1}\left[x_{i}\right]$ and $\Delta_{i-1}\left(h-h^{\prime}\right) \leq l$, where $\left(f_{i}\right)$ denotes the ideal of $R_{i}$ generated by $f_{i}$ and $u_{i}=\delta f_{i}$. By the induction assumption there exist integers $e_{1}, \ldots, e_{i-1} \geq 0$ such that $\left(h-h^{\prime}\right)\left(R a_{i-1}\right)^{e_{i-1}} \ldots\left(R a_{1}\right)^{e_{1}}$ $\subseteq Q\left[x_{1}, \ldots, x_{i}\right]+(f)_{i}$, for every such a diference $h-h^{\prime}$. Since $h^{\prime} \in(f)_{i}$ we obtain $h\left(R a_{i-1}\right)^{e_{i-1}} \ldots\left(R a_{1}\right)^{e_{1}} \subseteq Q\left[x_{1}, \ldots, x_{i}\right]+(f)_{i}$. Hence

$$
C\left(R a_{i}\right)^{m-u_{i}+1}\left(R a_{i-1}\right)^{e_{i-1}} \ldots\left(R a_{1}\right)^{e_{1}} \subseteq Q\left[x_{1}, \ldots, x_{i}\right]+(f)_{i},
$$

and the proof is complete.

Definition 2.4. Let $f=\left(f_{1}, \ldots, f_{n}\right) \in \mathcal{F}$. We say that $f$ is completely irreducible $(\bmod Q)$ if $f_{i+1}$ is $\Gamma_{P_{i}}$-completely irreducible as a polynomial in $x_{i+1}$, for every $0 \leq i \leq n-1$, where $P_{0}=Q$ and $P_{i}=\left[P_{i-1}, f_{i}\right]$.

Note that if $f$ is completely irreducible $(\bmod Q)$, then $f_{1} \in \Gamma_{Q}$ is $\Gamma_{Q}$-completely irreducible as a polynomial in $R_{1}$ and so $P_{1}=\left[Q, f_{1}\right]$ is a prime ideal of $R_{1}$. It follows by induction that $P_{i}$ is prime, for every $i \leq n$. 
By the construction in Theorem 2.3 and the results of Section 1, the sequence $f \in \mathcal{F}$ such that $P=\left[Q, f_{1}, \ldots, f_{n}\right]$ is completely irreducible $(\bmod Q)$. Therefore, for every prime ideal $P$ of $S$ with $P \cap R=Q$ there exists a sequence in $\mathcal{F}$ which is completely irreducible $(\bmod Q)$ and which determines $P$. The converse is also true.

Theorem 2.5. Let $Q$ be a prime ideal of $R$ and $\left(f_{1}, \ldots, f_{n}\right) \in \mathcal{F}$ a completely irreducible sequence $(\bmod Q)$. Then $P=\left[Q, f_{1}, \ldots, f_{n}\right]$ is a prime ideal of $S$ such that $P \cap R=Q$.

Proof. By Definition 2.4, $P_{i}=\left[P_{i-1}, f_{i}\right]$ is a prime ideal of $R_{i}$ with $P_{i} \cap R_{i-1}=P_{i-1}$, for $1 \leq i \leq n$, where $R_{0}=R$ and $P_{0}=Q$. So $P=P_{n}$ is a prime ideal of $S$ with $P \cap R_{i}=P_{i}$, for $1 \leq i \leq n$. Also, by Lemma 1.1, $f_{i} \in P_{i}$ is either zero or a polynomial of minimal degree in $P_{i}$ with respect to the condition $a_{i} \notin P_{i-1}$, according to $P_{i}=P_{i-1}\left[x_{i}\right]$ or $P_{i} \supset P_{i-1}\left[x_{i}\right]$, respectively. It follows as in Theorem 2.3 that $P=\left[Q, f_{1}, \ldots, f_{n}\right]$.

Denote by $\mathcal{I}$ the set of all the sequences $\left(Q, f_{1}, \ldots, f_{n}\right)$, where $Q$ is a prime ideal of $R$ and $\left(f_{1}, \ldots, f_{n}\right) \in \mathcal{F}$ is a completely irreducible sequence $(\bmod Q)$. Two sequences $\left(Q, f_{1}, \ldots, f_{n}\right)$ and $\left(L, h_{1}, \ldots, h_{n}\right)$ of $\mathcal{I}$ are said to be equivalent if and only if $Q=L$ and $\left(P_{i-1}, f_{i}\right) \sim\left(P_{i-1}^{\prime}, h_{i}\right)$ in the sense defined in Section 1 , for every $1 \leq i \leq n$, where $P_{0}=P_{0}^{\prime}=Q, P_{i-1}=\left[P_{i-2}, f_{i-1}\right]$, and $P_{i-1}^{\prime}=\left[P_{i-2}^{\prime}, h_{i-1}\right]$, for $i \geq 2$. It is clear that $\left(Q, f_{1}, \ldots, f_{n}\right) \sim\left(L, h_{1}, \ldots, h_{n}\right)$ if and only if $\left[Q, f_{1}, \ldots, f_{n}\right]=\left[L, h_{1}, \ldots, h_{n}\right]$. Therefore we have the following.

Theorem 2.6. There is a one-to-one correspondence between the set of all the prime ideals of $S$ and the set of all the equivalence classes of $\mathcal{I}$ module the equivalence relation $\sim$.

Remark 2.7. Perhaps is useful to point out that if $R=K$ is a field, then for every prime ideal $P$ of $S=K\left[x_{1}, \ldots, x_{n}\right]$ there exists a sequence $\left(f_{1}, \ldots, f_{n}\right)$ of polynomials in $P$ such that $P=\left[f_{1}, \ldots, f_{n}\right]$, where

$$
\left[f_{1}, \ldots, f_{n}\right]=\left\{g \in S: \text { there exist } e_{1}, \ldots, e_{n} \geq 0 \text { with } g a_{1}^{e_{1}} \ldots a_{n}^{e_{n}} \subseteq P\right\} .
$$

It should be convenient to find an algorithm to determine 0-completely irreducible sequences in this case.

Example 2.8. Assume that $R$ is a ring and $Q$ is a prime ideal of $R$. Let $f_{1}$ be a polynomial of degree one in $Z(R)\left[x_{1}\right]$ with $l c\left(f_{1}\right) \notin Q$, where $Z(R)$ denotes the center of $R$. Then $f_{1}$ is a polynomial in $\Gamma_{Q}$ which is $\Gamma_{Q}$-completely irreducible. Thus $P_{1}=\left[Q, f_{1}\right]$ is a prime ideal of $R\left[x_{1}\right]$. Take a polynomial $f_{2}$ of degree one in $Z(R)\left[x_{1}, x_{2}\right]$ such that its leading coefficient with respect to $x_{2}$ is not in $P_{1}$. Repeating the argument we determine a completely irreducible sequence $(\bmod Q)$ $\left(f_{1}, \ldots, f_{n}\right)$ in $R\left[x_{1}, \ldots, x_{n}\right]$ and a prime ideal $P=\left[Q, f_{1}, \ldots, f_{n}\right]$.

We give a concrete example (cf., [3], Example 4.1). Let $R$ be the integral domain of all the power series of $\mathbf{Q}[[t]]$ having the coefficient of $t$ equal to zero. Consider the polynomials $f_{i}=t^{2} x_{i}-t^{i+2}, 1 \leq i \leq n$. Then $P=\left[0, f_{1}, \ldots, f_{n}\right]$ is a prime ideal of $R\left[x_{1}, \ldots, x_{n}\right]$ which is not generated by the polynomials $f_{1}, \ldots, f_{n}$ (in general, if every $f_{i}$ is monic, then $\left[Q, f_{1}, \ldots, f_{n}\right]$ equals the ideal $Q[x]+\left(f_{1}, \ldots, f_{n}\right)$ generated by $Q$ and $\left.\left\{f_{1}, \ldots, f_{n}\right\}\right)$. 
Remark 2.9. The method given in this paper can easily be extended to describe prime ideals of polynomial rings in any number of indeterminates. In fact, choosing a well order for the indeterminates we can proceed by transfinite induction. Thus prime ideals will be described by sequences corresponding to the number of indeterminates. Finally, since every centred extension of a ring $R$ which has a commuting centralizing generator set is a factor ring of a polynomial ring over $R$, this gives also a description of prime ideals in this kind of centred extensions.

\section{ACKNOWLEDGEMENT}

This paper was written while the author visited the Department of Mathematics of University of Southern California. The author is grateful for the hospitality given by the members of the Department. He is also grateful to the referee for suggestions that helped to improve the presentation of the paper.

\section{REFERENCES}

1. D. Eisenbud and E. Graham Evans, Jr., Every Algebraic Set in $n$-Space is the Intersection of n Hypersufaces, Inventiones Math. 19 (1973), 107-112. MR 48:6125

2. M. Ferrero, Prime and Principal Closed Ideals in Polynomial Rings, J. Algebra 134 (1990), 45-59. MR 91h: 16008

3. M. Ferrero, Prime and Maximal Ideals in Polynomial Rings, Glasgow Math. J. 37 (1995), 351-362.

4. I. Kaplansky, Commutative Rings, The University of Chicago Press, Chicago (1974). MR 49:10674

Instituto de Matemática, Universidade Federal do Rio Grande do Sul, 91509-900, Porto Alegre, Brazil

E-mail address: Ferrero@if.ufrgs.br 